\title{
AWARENESS OF BIG DATA CONCEPT IN THE DOMINICAN REPUBLIC CONSTRUCTION INDUSTRY: AN EMPIRICAL STUDY
}

\author{
Paola F. Reyes-Veras, Suresh Renukappa and Subashini Suresh \\ Faculty of Science and Engineering, University of Wolverhampton, U.K.
}

\begin{abstract}
Purpose - The construction industry, being one of the main activities in the ever-demanding need for technology developments, sometimes falls short of other industries in terms of implementation. The adoption of Big Data (BD) in industries like health and retail has had positive impacts in aspects such as decision-making processes and forecasting trends that allows planning some future business movements. Hence, the question of whether these results can be recreated in the construction industry. Therefore, this paper addresses the level of awareness identified as a first step towards implementation of the BD concept within the construction industry in the Dominican Republic (DR).

Design/methodology/approach - Since little to no information exist on the subject, the selected approach to perform this research was qualitative methodology, twenty-one semi-structured interviews were studied using situational awareness. Four levels of awareness were developed based on the Endsley situation awareness model. Findings - The results showed that nearly ninety-five per cent of the interviewees had either no knowledge or very basic awareness of the BD requirements or intermediate awareness but only five per cent had applied BD concepts in the construction industry.

Originality/value - This paper provides an exploration of the level of awareness of BD in the DR construction industry and delivers evidence for the need to provide continuous professional development programmes for construction professionals and a need for an update of the curriculum in construction-related education.
\end{abstract}

Keywords: Awareness, Big Data, Construction, Dominican Republic, Technology, volume, and velocity.

Paper Type: Research Paper

\section{Introduction}

Big Data is one of the emerging terms with more presence in today's technological society, several authors such as Wang et. al., (2019), and Tamiminia et. al., (2020) have reported applications in various areas of science and engineering. The adoption of Big Data (BD) analysis in business and human resources management has had positive impacts in terms of improvement of the decision-making processes (Rabhi et. al., 2019). Big Data works as a solution for the "what to do" with the ever-growing volume of information that is produced every second, and it has applications across business sectors.

After financial services, construction industry generates the largest amount of data when compared to others. A key challenge for the profession is how to use this data to improve what is delivered. Despite the promise of artificial intelligence (AI) and the Internet of Things (IoT) industry, estimates suggest that up to $95 \%$ of construction industry data is not used but wasted (RICS, 2020). Khan (2008), Dlamini (2012), and RICS (2020) noted that the construction industry plays an important part in the economic growth of the countries. It is indeed one of the main characters in resources consumption and thus the ever-demanding need for technology developments. Being that the case, it sometimes falls short of other industries in terms of the implementation of technological developments. Construction methods have barely changed through the history of the industry, while access to resources and materials has varied significantly in recent years creating the need for change in the management and use of these resources. Technologies such as Building Information Modelling (BIM) promotes a more digitally integrated project management system. This has gained popularity recently since BIM allows to visualize and plan the development of construction projects more efficiently (Sarkar and Modi, 2015; Kamunda et al., 2020). RICS (2017) published an insight paper in conjunction with Oxford University on Big Data, smart cities, intelligent buildings, surveying in a digital world to equip construction professionals with the insights and tools to be able to consider these issues carefully and act with integrity in an increasingly data-driven world. 
DOI: $10.1108 / C I-05-2021-0090$

Some industries possess a higher success rate in adopting technological developments than others, e.g. the construction industry, which requires more time to make the changes that allow them to keep up with the latest technologies (Silverio-Fernandez et. al., 2020). In the same way, many industries have proven the benefits of adopting Big Data, hence the question arises as to whether these positive results can be replicated in the construction industry. Ngo et. al., (2020), explains that the adoption of BD in construction could produce great value, but there are no tools to assess the adoption capacity of construction companies.

This research seeks to explore the awareness and understanding of Big Data in the Dominican Republic (DR) construction industry which will serve as a first step to assess if basic measures such as education and popularization of the concept are required before an adoption plan can be developed. Dominican Republic is one of the fastest-growing economies in the region in the last 25 years, standing out as one of the few countries in the world that presented economic growth during the year 2020 which was affected by the COVID-19 pandemic (The World Bank, 2021). At least, 12\% of the country's gross domestic product is contributed by the construction industry, which constitutes one of the main economic activities (Central Bank of the Dominican Republic, 2020). Research studies by Silverio-Fernandez (2019) and Silverio Rodriguez, (2020) proved the willingness by the construction industry in DR to explore and use BIM and smart devices but there is no empirical research on Big Data which is the core rationale of this study.

A review of literature was initially conducted to identify the motivation behind the adoption of Big Data in other industries and to create a comparison between the practices in DR's industry and those presented in the literature. This also served as the basis for formulating the questions used in the data collection through semi-structured interviews, since a data collection method was needed that allowed collection of "open-ended data" which delivers rich, relevant data for the study and the exploration of interviewees thoughts (DeJonckheere and Vaughn, 2019). The participants were decision-making representatives of companies that play a leading role in the DR construction industry. The analysis of data was then performed using situational awareness analysis, which was used to classify the participants level of awareness of the Big Data concept and its characteristics through an adaptation of Endsley's model of Situation Awareness. The results of this analysis are presented and discussed in their respective sections, followed by the conclusion, but prior to it a theoretical background is presented.

\section{Theoretical background}

The first documented use of the term Big Data (BD) goes back to the mid-1990s (Li et al., 2016). Big Data is used to define the big amount of information contained in a database, which due to its heterogeneous characteristics makes it difficult to manage with traditional data analysis tools (Liu, 2015). In recent years, the term has regained popularity as a result of the increased production and transmission rate of information collected and handled thus gaining the attention of many disciplines and industries to take advantage of the benefits of its implementation (Tamiminia et al., 2020). Many studies (Caesarius and Hohenthal, 2018;Pigni, et. al., 2016; The Economist, 2012) agree that the adoption of BD results in positive outcomes such as improved financial performance, business optimization and innovation. Raguseo (2020) work highlights the "high revenue" promise as the main driver.

The emerging tools, software's and gadgets related to different disciplines promote the adoption of new technologies (Wang et al., 2020). In some countries, the adoption of technological developments such as BD occurs more naturally than in others. Hence dividing these tendencies into two groups. The first group in most cases adopt the new technology due to government support or incentives or due to industry initiatives, and that as a result exposing the benefits and challenges of adoption. The second group which implements the new technology after adopting the "wait and see policy" identifying the best practices and lesson learnt from the first group are also in pursuit of achieving the same benefits. Still, in both cases, it can be found that some industries are more prone to change than others, and some technological developments can be more easily adopted in some areas than others. The ease with which a discipline or industry adopts a new technology is primarily given by the type of companies that make up that industry. In this case, industries, where most of their companies are data-driven or carried out their operations digitally, are more likely to embrace the adoption of new technologies (Caesarius and Hohenthal, 2017, RICS 2020).

\subsection{Big Data Industries Applications}

The technological development and exponential growth of information have created the perfect environment for the proliferation of tools that, as Big Data, allow to extract useful insights from this data, becoming a determining factor in the decision-making process (Wang et. al. 2020). 


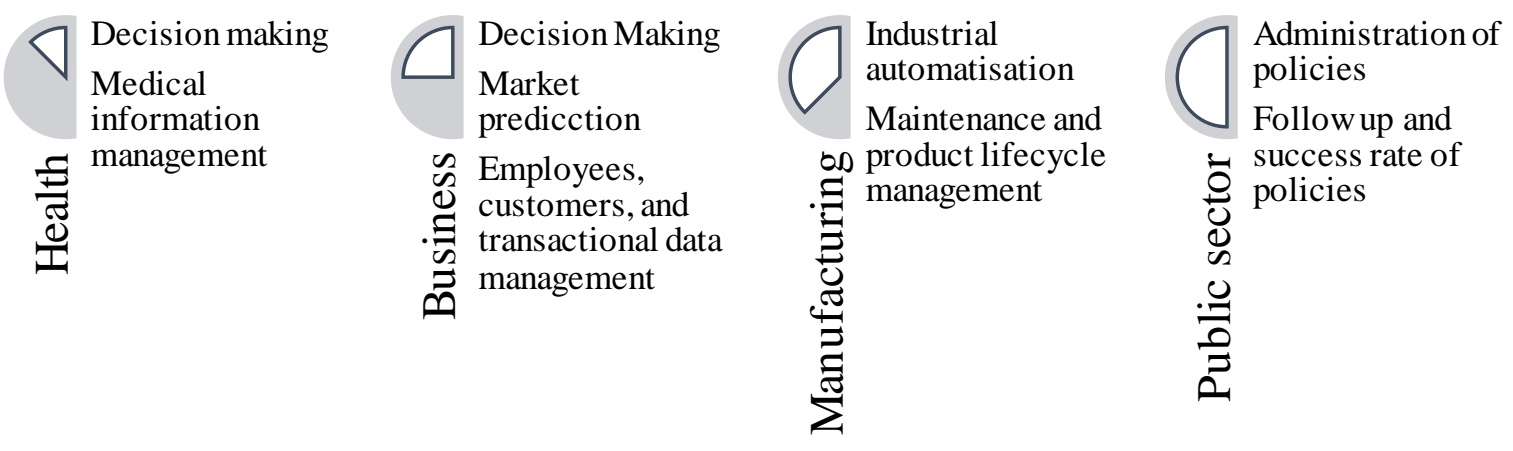

Figure 1: Big Data applications in different industries

As shown in Error! Reference source not found., fields such as Healthcare, Public Sector Administration, Business, Manufacturing, among others have taken advantage of the Big Data development wave present in the last couple of years (Chen et. al., 2020, Rabhi et. al., 2019; Tabesh, et. al., 2019). Many fields have adopted Big Data to substitute their outdated systems for data management in order to provide better management and analysis of the high volumes of data they now produce and possess (Boyd and Crawford, 2012). Tamiminia et. al., (2020), agrees by confirming that the massive volumes of existing geospatial data, its variety of origins and formats, and growing diversity and accessibility, demonstrating how very different fields are motivated by the same aspects in terms of BD adoption. For instance, Chen et. al., (2020) states that the increasing amount of patient data in healthcare formed by information assets with high volume, velocity, value, and diversity through healthcare data resources. The BD based healthcare information system has been growing rapidly in recent years and is being adapted to derive important health trends and support timely preventive care. Mainly, the increasing amount of patient data can no longer be handled by the traditional approaches. Chen et. al., (2020), noted that Electronic healthcare records (EHRs), which use big data analytics for major evaluations of diseases and performance of epidemiological analyses. Finally, the decision-making aspect plays a major role in the healthcare industry, therefore the data that propels this process needs to be managed with an appropriate tool such as BD.

Other areas such as business and management rely upon a subjective judgement for decision-making. However, according to Rabhi et. al., (2019) increasingly, important decisions are on hard data, which can be obtained through big data analytics. Also, in Human Resources Management, the rate of adoption of Big Data are due to the need to manage the large volume of employees, customers, and transactional data in organisations, with the purpose of helping companies to "make decisions and create bigger benefits". Wang et. al., (2020) acknowledges that BD application in the manufacturing industrial field is still in its infancy but at the same time, mentions its application in several fields such as natural resource management, industrial automatization and maintenance and Product Lifecycle Management, which are already being developed and applied. Another series of most common applications are presented by Tabesh, et. al., 2019, in their study of BD implementation strategies from the administrative point of view (Error! Reference source not found.). 

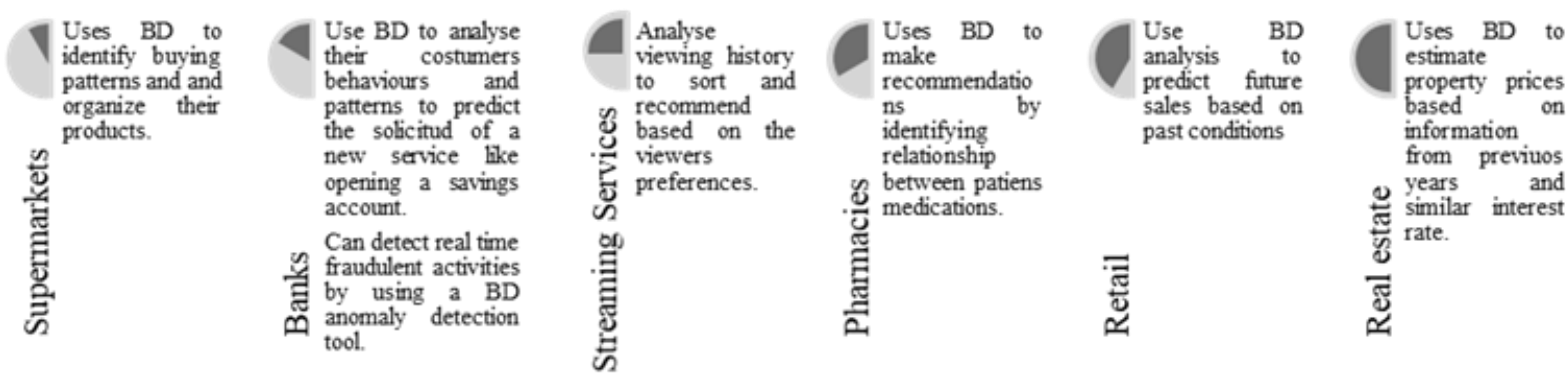

Figure 2: Extract form table "An overview of important Big Data analytics algorithms and applications".

Source: Tabesh, et. al., 2019

Big Data has garnered an incredible amount of attention in recent years, this topic has become the focus of conferences and scientific publications all around the world, thus becoming a major innovation source for academia, causing even a widespread proliferation of research around this topic ( $\mathrm{Li}$, et. al., 2016). Contrary to popular belief that $\mathrm{BD}$ is technology-oriented to private industry, the public sector has also started to benefit from its adoption. Khurshid et. al., (2019) indicates that public bodies are one of the producers of data in large quantities such as geospatial maps, public records of transfers, appointments, financial statements, census data, and environmental datasets. Governments have been actively making available to the public large amounts of data without restrictions creating Big Open Data (BOD) a subcategory of BD that shares its same basic characteristics. Among the applications of this BOD, businesses can use this data for commercial purposes, it can allow the public to make more informed decisions and the most important thing is that it will provide real-time data for policymaking to deal with intricate situations. Also, the cyclic process of BD analysis (Figure 3) (Rabhi et. al., 2019), makes it an ideal tool to follow up on the implementation of those public policies. Khurshid et. al., (2019) also highlight the fact there has been an increase in government uses, for example, Pakistan has adopted the use of BOD, based on the need for transparency, as a follow up of the recent technological developments and development of social and commercial value, among others.

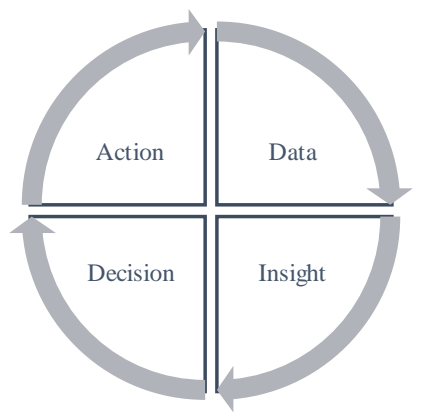

Figure 3: Big Data analysis process

\subsection{Big Data in Construction}

Little is known about the adoption of BD in construction since the developments that make possible its use in this industry are still in the early stages (Ngo, et. al., 2020). Still, there is evidence indicating that its adoption within the industry would produce similar results to those obtained in other disciplines. For instance, Neilson et. al., (2019), indicated that traffic data complies with all requirements needed to be considered as BD, the information from monitoring equipment and wireless sensors that have been installed to promote safety and traffic monitoring 
are already being used to gain insights that promotes a more efficient traffic management environment. It also highlights that the projected future of transportation which is the case of autonomous vehicles will depend on realtime traffic data that BD could help to deliver more effectively. In the same line, Li, et. al., (2019), also consider transport as a potential beneficiary of BD adoption, as well as other aspects, directly and indirectly, related to construction such as monitoring of critical infrastructures and geospatial fields which are used to better locate construction projects (Filipović, et. al., 2020).

Authors such as Bilal, et. al. (2016) and Oudjehane, et. al., (2017), have identified the source of the data available within the construction industry to be considered BD in the form of project schedules, reports, drawings, site images and sensor data as well as information and designs coming from the us e of BIM and the use of sensors and technologies related to Io T. In a more recent study, Ngo, et. al., (2020) listed the applications of BD in relationship to the project life cycle (Error! Reference source not found.), also mentioning the general characteristic of supporting the decision-making processes, which is considered as a general characteristic through all fields.

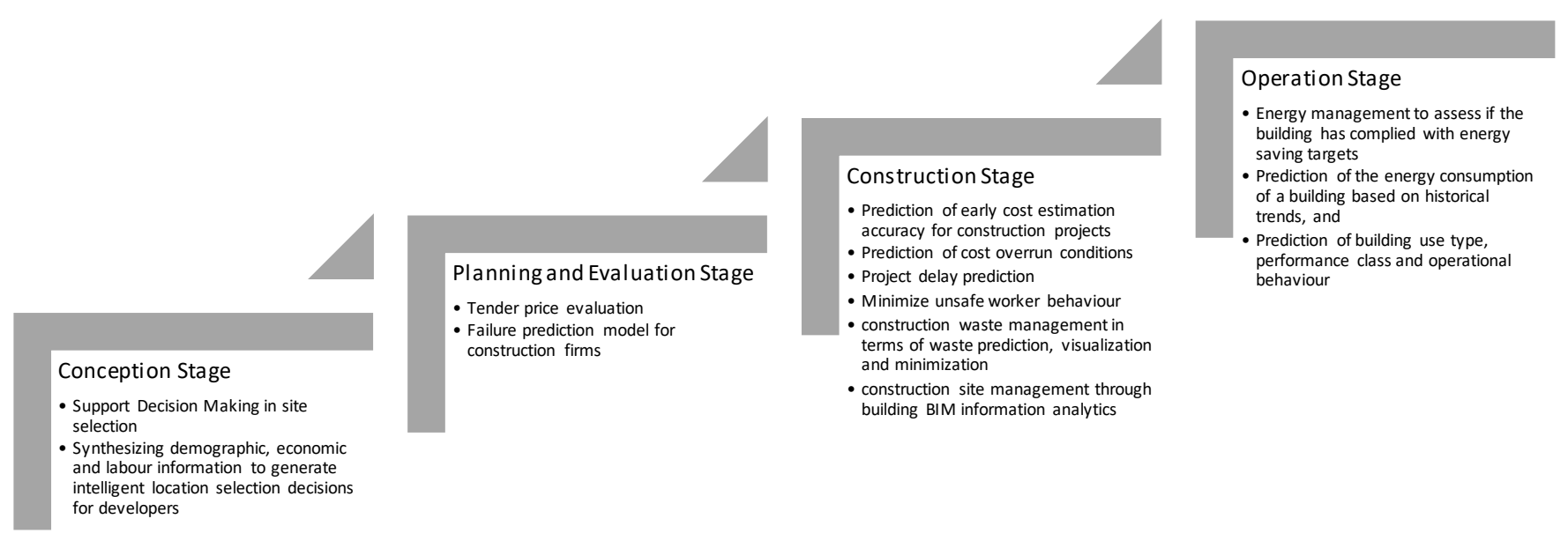

Figure 4: Applications of Big Data in the Construction Project Lifecycle

More recently, the development of strategies that require the participation of the construction industry such as smart cities whose objective is "to increase the quality of life in multiple dimensions, such as health, transport, energy efficiency, etc."Naoui et. al., (2020) and Saya et. al., (2020) noted that BD is used to manage and analyse its database collected from numerous and diverse sources, in huge quantities and most cases in real-time. Activities such as the reduction of energy consumption are one of the main goals of governments and a focal point of both the construction industry, the expansion of smart cities and sustainability, which can be achieved by the application of BD by allowing the cooperation of various technologies that together can provide energy efficiency than each technology in its own.

\subsection{Construction Industry of the Dominican Republic}

Just as in the rest of the world, the construction industry plays an important role within Dominican Republic's economy, as stated before, the industry contributes $12 \%$ of the gross domestic product in the form of job and income generation, according to the Central Bank of the Dominican Republic (2020). The natural phenomena such as earthquakes and the cyclonic season to which the island is subjected during the year require that the infrastructure be able to withstand the effects of these phenomena placing a special level of responsibility on the Dominican construction industry. The execution of both public and private construction projects with the view to meeting needs in areas such as housing, tourism, and rural development, make this industry one of the main players in the Dominican economy (Silverio-Fernandez, et. al., 2019).

The construction industry is intimately linked to the economic development of the Dominican Republic, from the generation of jobs to the infrastructural development of the nation, such as the improvement of the quality of life through low-cost housing, infrastructure linked to basic necessity services and other factors exposed in the national development strategy (MEPYD, 2017).

Undoubtedly, the construction industry of the Dominican Republic could benefit in a general way from the implementation of Big Data, but it could also specifically aid with the fulfilment of the goal of enhancing the scope 
DOI: 10.1108/CI-05-2021-0090

of sustainability in the industry in general that comes aligned with the country's commitment to meet the 2030 sustainable development goals through the national development strategy mentioned above which even includes specific points to be applied in this industry. This could be achieved by adopting BD applications such as:

- The overall efficiency of the activity by analysing data from previous projects and identifying errors that can be prevented in future projects.

- Weather and traffic data analysis for construction planning.

- Smart water meter data analysis to identify new locations for wastewater treatment plants enhancing effectiveness.

- Governmental database for construction waste management and reduction.

- $\quad$ Tourism data analysis to identify new areas for resort development.

\subsection{Measurement of Awareness}

To "measure" the awareness of BD in DR, Endsley's "Theory of Situation Awareness" was adopted for this study. The most generally accepted interpretation of situation awareness, is defined as the "... perception of the elements in the environment within a volume of time and space, the comprehension of their meaning, and the projection of their status in the near future" (Endsley, 1995). Based on this model, the Situation Awareness Global Assessment technique, which according to Dishman, et. al., (2020), considered "the only direct and objective situation awareness measurement tool". Situational awareness in Endsley's model has three (3) levels (Error! Reference source not found.): perception, comprehension, and projection (Endsley, 2014). For this study, an adaptation of the Endsley Model was created (Error! Reference source not found.). Level zero (0) of "No Awareness" was

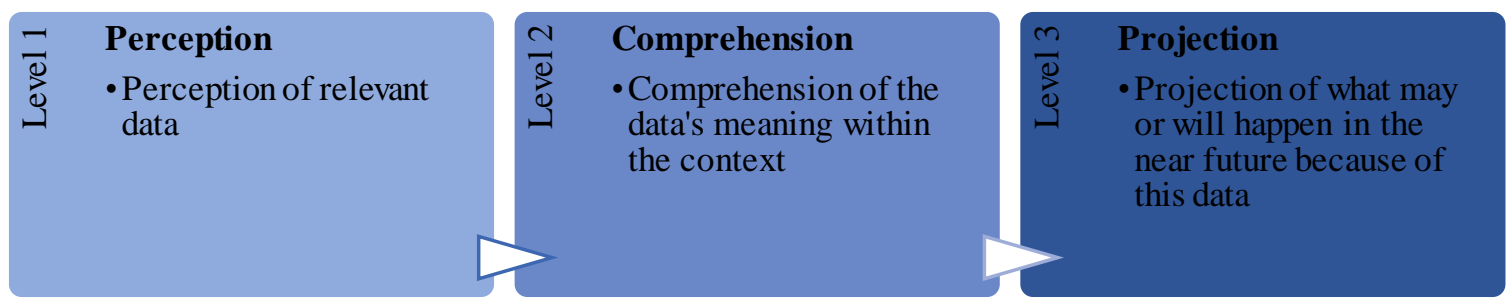

Figure 5: Endsley original model of Situation Awareness added.
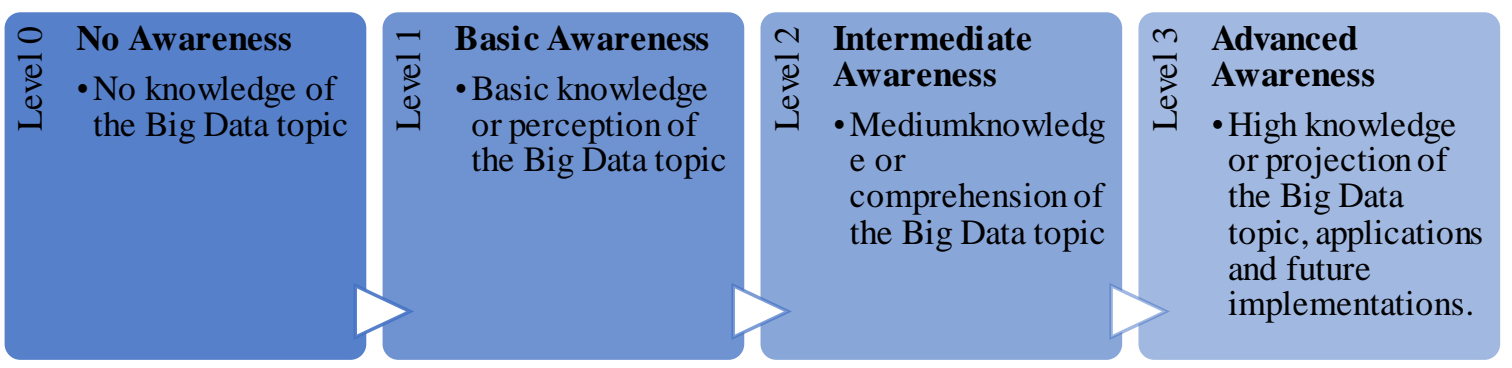

Figure 4: Endsley's Model Adaptation

The concept of Situation Awareness (SA) was initially used in military and aviation applications (Endsley, 1995). Over the years the application of the SA is seen across several industries to provide an awareness classification. For instance, disciplines such as health have used Endsley's model of SA in mental health (Moura, et. al., 2020). Also, for thoracic surgery, obstetrics, nursing and anaesthesia (Dishman, et. al., 2020). Likewise, the program evaluation field used SA to help improve the decision-making skills of program evaluators (Marson, 2020). Manufacturing has also used SA analysis to reduce the incidence of accidents related to forklift driving (Choi, et. al., 2020). However, there is no evidence of this model been applied to Big Data and in particular to the construction industry. 


\section{Research scope and gap}

The first step in understanding the factors that could enable a future adoption of Big Data in construction is to identify the state of knowledge that is possessed on the subject, which is the scope of this study. It will help to understand or identify if the need exists for certain goals such as concept popularisation or a wider understanding of the characteristics is required before an adoption plan can be developed, in other words, this is an initial study that comprises only the general knowledge aspects of the investigation. To explore and understand the critical aspects for BD implementation and a path to adopt, there should be first an industry-wide awareness of the technology.

Representatives from medium and large construction companies in the Dominican Republic were interviewed to assess their level of awareness with questions that explore their understanding of BD using topics related to the concept, characteristics, benefits and data handling. This study is limited to analysing concepts related to general knowledge about big data, leaving other aspects also important for implementation such as barriers, challenges, key drivers, etc., for future research.

Even when there is literature that supports the implementation of Big Data in construction, it recognizes that it is still in its initial stages and that a clear path to adoption has not yet been documented (Ngo, et. al., 2020). No relevant results were found of the Dominican Republic's strategies to implement new technologies apart from (Silverio-Fernandez, et. al., 2019 and 2020) but the studies are regarding smart devices. Therefore, this study seeks to explore the research question: What is the level of awareness about Big Data and its characteristics within the Dominican Republic's construction industry?

This study provides an insight into like-minded countries within the region that is like the Dominican Republic as the DR plays a major role in the Latin American and Caribbean region with one of the strongest economic growth according to the World Bank (2020), which is the reason why any study carried out in DR could identify a path for other countries in the region.

\section{Research methodology}

Big Data is a relatively new concept for society in general, even more new, for some industries such as Construction, studies that explore the adoption of BD in this industry are scarce and mostlybased in countries with great technological advantage and non-existent in countries like the Dominican Republic. Hence the methodology adopted for this exploration was dictated by data availability (Kumar 2014), since little to no information exists on the subject, the selected approach to perform this research was qualitative in nature. Furthermore, the data collection method was dictated by the exploratory nature of the research and the need to collect reliable and comparable data while also allowing the participants to freely share their points of view, for which, the semistructured interview was the perfect tool (Cohen and Crabtree, 2006). This data collection method also allowed the collection of "open-ended data" required by this type of studies which delivers rich, relevant data and the exploration of interviewees thoughts (DeJonckheere and Vaughn 2019). In-depth data required prevented other methods such as questionnaires, participant observation and even structured interviews to serve as a suitable option (Silverio-Fernandez et. al., 2020).

So far, the information and literature accessible about the adoption of BD are more common in some areas such as health, statistics and economy, and excludes others in which this technology is considered emerging like the construction industry. Hence the need to comprehend the understanding of decision-makers about BD and its basic characteristics within the industry, to establish a knowledge baseline that allows tracing a future route for adoption. This resulted in the creation of the general knowledge sectionaddressed in this paper, conformed of four questions, which was designed to probe the understanding of the BD concept and its characteristics. The first question explores the knowledge of the participants about the concept of BD and its relationship with the construction industry, which is driven by the fragmented discourse and lack of homogeneity on what constitutes the technology definition (Gandomi and Haider, 2015). The second question arose from the need to explore the understanding of the participants about basic characteristics which make up BD and its relationship with the construction industry. The third question was set to determine whether the participant companies had any level of implementation of the technology or in its place any general protocol that could enable a future implementation. The final question of the section enquired about the knowledge of the participants of any other BD implementation outside construction which could potentially enable a better understanding of the technology's prospects within construction. 
Before exploring and understanding what the critical aspects for BD implementation are, it is necessary to establish and assess the level of knowledge and subsequently generate the pertinent recommendations to ensure that the industry has the necessary tools to understand what is being discussed and thus prepare for future implementation. Ultimately, twenty-one (21) semi-structured interviews were carried out to explore different areas concerning Big Data in the DR Construction industry.

\subsection{Sampling Process}

The interviews were directed at large and medium-sized companies with an undeniable presence within the productive market and whose representatives (interviewees) were top-level executives involved in their decisionmaking process. The table below (Table 1) shows the positions to which the interview participants belong.

Table 1: Breakdown of professionals participated in

\begin{tabular}{lc}
\hline Position & Number of Interviewees \\
\hline Chief Executive Officer (CEO) & 1 \\
\hline Vice-President of Operations & 1 \\
\hline Superintendents & 2 \\
\hline Seniors & 4 \\
\hline Managers & 10 \\
\hline Coordinators & 3 \\
\hline Total & 21 \\
\hline
\end{tabular}

The participated companies were all construction-oriented companies with no obvious focus in a specific business area, meaning that each company is dedicated to developing any kind of infrastructure projects such as residential buildings, transport links, resorts and so on.

Moreover, the literature showed that the size of the company influences their ability to implement newtechnologies such as BD, where the bigger the company the most likely it can possess the investment capability and accumulate the amount of data necessary to implement a successful BD analysis (Ngo, et. al., 2020), which was considered at the time of filtering the companies that would be contacted to participate in the study. This literature-based factor defined the sampling technique as non-probabilistic, which allows the selection of the sample based on certain criteria (McCombes, 2019). In this case, providing a group of characteristics that both companies and participants should meet to be considered suitable for this study. The size of the company and the position of involvement in the decision-making process of the interviewee were the factors considered when establishing the sample. Moreover, the national taxpayer registry of the Dominican Republic was employed to identify the construction companies with the potential to participate in this research.

From the database mentioned above, the participating construction companies were selected following the classification according to size provided by the laws of the Dominican Republic (National Congress of the Dominican Republic, 2008), this indicates that the organizations will be classified as:

- Micro: with 1-15 employees, less than RD\$3,000,000.00 in active capital or less than RD\$6,000,000.00 in annual revenue.

- Small: between 16-60 employees, between RD\$3,000,000.01-12,000,000.00 in active capital or between RD\$6,000,000.01-40,000,000.00 in annual revenue.

- Medium: between 61-200 employees, between RD\$12,000,000.01-40,000,000.00 in active capital or between $\mathrm{RD} \$ 40,000,000.01-150,000,000.00$ in annual revenue.

- Large: with more than 200, more than RD\$40,000,000.00 in active capital or more than $\mathrm{RD} \$ 150,000,000.00$ in annual revenue.

Due to their enhanced capability for technology investment (Ngo, et. al., 2020), only large companies were considered for this study. The sampling process started with a first stage in which a telephone call was made explaining the scope and purpose of the research and seeking potential companies their willingness to participate, followed by the second stage of requesting those who answered the first call, to nominate one or more individuals in their organisations that meet the criteria and would be interested in sitting down for an interview. The third stage consisted of contacting the nominated participants and arranging the details for the interviews to take place, and 
DOI: $10.1108 /$ CI-05-2021-0090

finally, the fourth stage consisted of the actual interview with the participants. The detailed presentation of the sampling process can be observed below (Table 2).

Table 2: Pre-Interviewing Process

\begin{tabular}{ccc}
\hline Stage I & $\mathbf{7 8}$ & Companies Contacted \\
\hline & 38 & Answered \\
\hline & 40 & Not answered \\
\hline Stage II & $\mathbf{3 8}$ & Answered \\
\hline & 19 & Interested \\
\hline & 15 & Maybe interested \\
\hline Stage III & $\mathbf{3 4}$ & Not interested \\
\hline & 26 & Answered \\
\hline & 1 & Maybe to participate \\
\hline & 7 & No, to participate \\
\hline Stage IV & $\mathbf{2 7}$ & Answered \\
\hline & 19 & Did Participate \\
\hline & 8 & Not Participate \\
\hline
\end{tabular}

A total of seventy-eight (78) companies were contacted and asked whether they would be willing to participate in the investigation, a primary response rate of forty-eight-point seven per cent (48.7\%) was obtained.

The pre-interview process can be divided into four (4) stages from the moment of the first contact to the interview. Stage I represents the total of contacted companies, from which thirty-eight (38) answers were received Stage II, represents the type of answer received about willingness to participate, nineteen (19) companies responded with a definite positive willingness to participate, fifteen (15) responded with non-definitive interest and the remaining four (4) companies expressed no willingness in participating in the study. Stage III represents where the criteriameeting participants were contacted to schedule the interview appointment, from which twenty-six (26) participants proceed with the scheduling, one asked to reconvene at a later time and the other seven (7) decided not to participate. Finally, Stage IV represents, the actual interviews carried of which ended up being twenty-one (21) from nineteen (19) companies.

To determine the number of interviews that would be used for the investigation, the saturation phenomenon was employed. Saturation is a well-established method to determine how many interviews are enough in a qualitative study. This term is used to identify the phenomenon where the interviews do not contribute any new or additional information to the investigation (Latham, 2020). Guest et. al., (2006), in their study, reached saturation point in the twelfth interview. In this study, the final sample consisted of twenty-one (21) interviews, from 19 companies. Crouch and McKenzie, (2006) state that less than 20 participants in qualitative research helps establish a close relationship and consequently a more reliable exchange of information, identifying the ideal number is between fifteen and twenty (15-20) participants.

Another aspect considered is data generalisation from which conclusions can be drawn from particular situations (Polit, and Beck, 2010). As explained earlier in section Error! Reference source not found. where is described that due to the economic growth of the Dominican Republic within the Latin America and Caribbean region, it is in an advantageous position and could serve as an example regarding the implementation of new technologies such as BD.

The data collection process lasted from October 2019 to March 2020 and were held in Santo Domingo, Dominican Republic. The interviews were captured using voice recordings and the duration was between nine (9) and twentytwo (22) minutes approximately. Each interview was transcribed and then translated into English from Spanish, which is the official language of the Dominican Republic, except one that was carried out in English with the prior approval of the interviewee. 


\subsection{Data Analysis}

The analysis method adopted is Awareness analysis. This study aims to explore the awareness and understanding of the participants about BD. An assessment of the level of awareness was performed covering key aspects such as concept, characteristics, benefits and data handling all in relation to the adoption of new technologies.

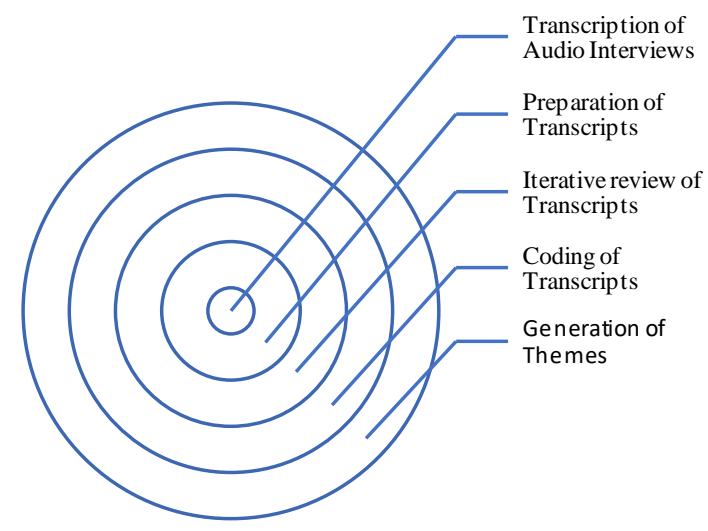

Figure 6: Creswell's DataAnalysis Process described in “Guide for Qualitative Data Analysis”

In preparation for the data analysis part of a process described by Creswell in its "Guide for Qualitative Data Analysis" (Error! Reference source not found.) was considered. Creswell (2013) describes five steps that were used to prepare the interviews for the analysis process. The three (3) first steps were applied to the data collected as preparation for the analysis.

The data analysis process of coding and generation of themes was performed using the program Microsoft Excel, each code was placed in a cell, thus creating a grid containing a classification according to the coding assigned to each question and each interview (Caufield, 2019). This classification represents the levels of awareness about Big Data within the Endsley model (Figure 6). Furthermore, the individual results according to each question are presented to visualize awareness in each specific area represented by the questions.

\section{Findings}

In this study, during face-to-face interviews, a total of four questions were asked in order to understand the awareness around the Big Data concept and its characteristics. In this segment, the findings obtained from the analysis of those four questions are presented.

\subsection{Concept Awareness and Relationship with the Construction}

To capture the level of awareness on the concept of "Big Data", a first question was raised, i.e., what is your level of awareness of the "Big Data" concept? Overall, the interviewee's awareness of the BD concept was distributed between various levels of situational awareness in the modified Endsley's model. Of the interviewees, 33\% (7 of the 21) noted that 'no awareness' or never heard of the Big Data concept and its relationship within the construction industry. This reflects on the no awareness Level 0 of Endsley's model. For instance, interviewee $\mathrm{I}_{8}$ highlighted that:

"No, I had not really heard the term until this moment".

It is evident from the above statement that, the DR construction organisations lack a basic understanding of the concept of Big Data. Therefore, to improve the DR construction industry competitiveness, decision-makers have to recognise and understand the concept of Big Data. It is worthwhile to consider a holistic impact of Big Data concepts to improve the project delivery processes of the DR construction industry.

$33 \%$ ( 7 of the 21 ) of the interviewees had 'basic awareness' of the concept of Big Data and are at Level 1. This reflects on the basic awareness Level 1 of Endsley's model. For instance, interviewee $\mathrm{I}_{20}$ highlighted that: 
DOI: $10.1108 /$ CI-05-2021-0090

"Very generally, a big database that can be used to identify trends and to manage information".

Similarly, I12 stated that:

'I've heard of the term applies to statistics and economy, but I never hear of any relationship of BD and Construction".

From the above, it is evident that there is a basic awareness of what BD is but not concerning the construction industry. Therefore, there is a need to raise awareness of Big Data concepts and applications in the context of the construction industry. It is evident that $66 \%$ of the interviewees ( 14 of the 21 ) lacks a basic understanding of the concept of $\mathrm{BD}$ and its relevance to the construction industry. Therefore, there is a need to develop a continuous professional development programme for the benefit of present construction professional in the DR.

Of the interviewees, $29 \%$ ( 6 of the 21 ) noted that they have an 'intermediate awareness' of the concept of Big Data. This reflects on the intermediate awareness Level 2 of Endsley's model. For example, $\mathrm{I}_{4}$ and $\mathrm{I}_{11}$ noted that:

"BD in relation with the construction industry is about the amount of data created in a construction project". - $\mathrm{I}_{4}$

"From what I understand is that this technology allows you to extract insights of data from past projects in order to make better decisions in the management of the future projects" $-\mathrm{I}_{11}$

It is evident that there is an intermediate level of awareness in terms of the applications of BD in the construction industry, but the interviewees were not involved in the implementation of the BD concept. Therefore, it is important to document and provide BD leadership awareness programmes that include the holistic approach for implementing BD in the construction industry.

Whereas just 5\% (1 of the 21 ) interviewees noted that they have an 'advanced awareness'. This reflects on the advanced awareness Level 3 of Endsley's model. The interviewee $\mathrm{I}_{5}$ noted that:

"I knew the term Big Data, actually last year because I used a software for data analytics as it is a big corporation, they have like two departments, one for construction and one that's like a supplier. The operation manager used information for the statistics of the sales which quarter of the year make the most XXXX, in comparisons with this year and the last year. As well as the construction department saw how they used the information. As a big corporation, more data is gathered on a continuous basis. Therefore, I had to learn what Big Data is and how to manage Big Data and apply it to provide trend analysis and enhance decision making”.

\subsection{Big Data Characteristics}

The second question explored the level of awareness around the BD defining characteristics and its relationship to the construction industry, i.e., Are you aware of the volume of data produced in a Construction Project and the variety of that data? In this case, more than half of participants showed evidence of belonging to the basic level of awareness within Endsley's model, while the rest was distributed between medium and no awareness with the highest per cent belonging to medium awareness,

$5 \%$ ( 1 out of 21 ) of the interviewees had no awareness or knowledge of the Big Data characteristics asked in question two, which places them within the No Awareness level (Level 0) of the model, as evidenced by the following comment from $\mathrm{I}_{16}$ :

"Well, we always try to have everything in physical form in each project, in the office we keep copies and files of everything but in site, we have everything printed, you know if someone needs to check something or you have supervision visiting, everything needs to be there. Well, we storage basically everything, from CAD blueprints to pdfs, to the payroll of the workers, etcetera".

The above shows that there is an existing part of the industry in the DR that is not yet integrated into project digitalization, the industry is still dependent on printed material to manage the projects on-site, demonstrating a 
DOI: $10.1108 /$ CI-05-2021-0090

clear need for understanding benefits such as transparency and reduction of errors that happen as a result of the digitized management of the projects and that technologies such as BD can provide.

Nevertheless, $57 \%$ (12 out of 21 ) of participants demonstrated basic awareness of the BD characteristics when questioned, for example, $\mathrm{I}_{15}$ and $\mathrm{I}_{19}$ agreed that:

'Well, I couldn't give you a specific number, but only between the different versions of the same documents, I mean, every time that we have to modify a blueprint lest say, we store all the previous versions, and that could take Gigabytes a single document, I don't know if you understand what I mean, and the variety has to do with all the different formats". I15

"I would say that I have an idea of how much is produced in a project, you mentioned things like, spreadsheets, blueprints, pictures, maps, etcetera". I19

These comments reveal that most professionals in the construction industry possess up to some extend understanding of the characteristics of BD in terms of volume and variety of data handled in a construction project, thus demonstrating basic awareness, but in the same way, is evident the need to further popularize the concept and its characteristics to create a general uniform understanding of the technology and how to benefit from it.

Finally, 38\% ( 8 out of the 21) participants showed medium awareness of the BD Characteristics, demonstrating a deeper understanding of the implications of the data generated in a construction project and how it relates to BD and relating to the "Intermediate Level of Awareness" in Endsley's Model. For instance, interviewee I 2 noted that:

"Eh, like tangibly no, but I do know because I work with that, I work data production, information production and transmission, now, I know that there is a background, like something that you cannot see, some invisible information that is also part of it that I don't really know to which magnitude it develops or grows on the back.".

The evidence shows that there is an intermediate level of awareness and understanding of the characteristics and that overall, participants have a good idea of the data produced in a construction project and how this data fits the Big Data technology, however, it also illustrates the need to provide the necessary tools and training needed to drive adoption.

In the case of BD characteristics, no interviewee expressed advanced awareness or high understanding of what they are and how they can be identified within the construction industry context thus reaffirming the need for professional training focused on the following of future industry developments which will allow that when they encounter the elements that currently serve as the basis for these technologies, they will know how to identify them and take advantage of their potential.

\subsection{Data Management Protocol}

After asking participants about the volume and type of data that they handle in their construction companies another question was asked to explore whether their organizations have in place any protocol of data management, their answers were distributed within Endsley's Model of situation awareness in the following way:

43\% (9 out 21 answers) of interviewees expressed not having any data management mechanism in place in their respective companies, which places them within level 0 of "No Awareness" in Endsley's model. The answers provided by interviewees $\mathrm{I}_{15}, \mathrm{I}_{19}$ and $\mathrm{I}_{21}$ show exemplify the two situations that were found in this case:

"Ahh well, we store it, while the project is running it stays in an open online server that way everyone involved can have access to it and then when the project is finished we back it up and store it in the cloud. Yes, we found that this is the best way to ensure that everyone can contribute and once you finish, either for an audit or just to keep as company records you need to have all the final documents safely storage". I 15

“Only storage”. $\mathrm{I}_{19}$

'No, I don’t think so". I 21

The first two comments ( $\mathrm{I}_{15}$ and $\mathrm{I}_{19}$ ) describe the situation in which the interviewees don't recognise data storage as a data management protocol, the action of storing digitized information either in the cloud or on a physical 
server conforms a data management process, and the third comment $\left(\mathrm{I}_{21}\right)$ depicts the situation in which the organization simply does not possess such protocol at all, in either case, the need to clarify the concept of BD and its implications is evident.

Another $43 \%$ of interviewees (9 out of 21 ) expressed to have some kind of data management protocol which in most cases was an online database with the information of previous and current projects. This recognition of the protocol is what has placed them at level 1 of "Basic Awareness" of Endsley's Model, which was evidenced by interviewee $\mathrm{I}_{10}$ in the following comments:

"Yes, but not in a structured way. We have received or well-integrated application proposals such as Aconex and PROCORE but we still do not see it feasible. Meanwhile, we handle everything from a protocol of folders in Dropbox".

In the same way, Interviewee $\mathrm{I}_{11}$ stated that:

"Yes, we use cloud storage, so all data is available at all times".

The previous statements show that the pressing need exists for the Dominican industry to adopt and relate to digital terms that will help open the doors of modernization, not only with the adoption of BD but also with the adoption of other predecessor technologies and simple protocols such as data gathering and data storage which in most cases is already being implemented without being identified as such.

$9 \%$ of the participants ( 2 out of 21 ) provided answers fitting to an intermediate awareness of data management protocol in their companies, with data gathering or project management software in place. In this matter, $\mathrm{I}_{1}$ comments that:

"Not, not actually, I mean, like I, like I said we used a software that already gathers the data, and we only worked with that data, but the pictures, the videos, or the, or the spreadsheets, people use in site, but we didn't do anything with it".

The percentage of companies that recognize and use some type of data management system is minimal, which shows the great need for understanding these tools and the benefits they can provide to increase efficiency and productivity.

Ultimately, only one participant representing the last 5\% had "Advance Awareness" or high comprehension in the issue of identification and implementation of data management or analysis tools. Interviewee $\mathrm{I}_{5}$ noted that:

'We use a management program: PMWeb; outstanding follow-up program: Asana. We also have protocols presented by the manager and owner of the company to maintain the organization of information between projects".

With the exception that it is a traditional data analysis system, interviewee I5 exemplifies a scenario where the company has identified the benefits of adopting a data management tool and has decided to invest in its development. Unfortunately, this case represents the minority of companies, but it could, in turn, serve as an example like a case study that could guide the industry in the near future, however, more cases like this are needed in order to generate confidence in this new technology.

\subsection{Awareness of implementation outside the construction industry}

The final question of the section explores the awareness of the participants into any other application of Big Data outside the construction industry, i. e., Are you aware of the benefits as a result of Big Data adoptions in other industries? Which yielded mixed responses distributed between the four levels of the modified Endsley's Model, in this case, most of the answers are almost evenly distributed between levels 0 and 1 and the remaining $30 \%$ between levels 2 and 3 .

$38 \%$ (8 out 21) interviewees expressed to have no awareness of any other application of Big Data outside the construction industry, this percentage is closely related to the $33 \%$ obtained for the same level in question number one, meaning that most of all the people and then some who did not know the term or its relationship with the construction, nor they have heard from it outside this scope. In this case, Interviewee $\mathrm{I}_{4}$ expressed that:

"No. nothing that I could mention specifically". 
DOI: $10.1108 /$ CI-05-2021-0090

This can be considered an indication of the early stage of development in which this technology is, meaning that the need to raise awareness of Big Data concepts and applications is not only in the context of the construction industry but in general since this can improve the general understanding around the technology and create momentum in the exploration of what can be achieved in the construction industry.

Of the interviewees, another 33\% (7 out of 21) expressed that they possess a basic awareness or perception of other Big Data applications outside the construction, which allocates them in Level 0 in Endsley's Model. In this case, most of the people had heard vaguely of the term in other fields rather than the construction industry. For example, I20 expressed that:

"Again, very vaguely I could say I've heard of its use in information management and in statistics to identify trends, I couldn't say more than that".

The great part of the Dominican construction industry that has very little or no knowledge about this technology indicates a lack of interest in modernization and follow-up of new technologies. It also reveals the need to foster professionals who lead the industry by being bold, accepting risks and adopting new technologies like this one that today is being established or explored in almost all other industries.

Moreover, only 19\% of interviewees (4 out of 21) expressed an intermediate level of awareness of Endsley's Model, detailing specific areas of implementation outside the industry. Very concisely Interviewee $\mathrm{I}_{11}$ expressed that:

"In other countries, they have been able to predict shopping tendencies and even drops in the Stock market using BD”.

Similarly, Interviewee $\mathrm{I}_{17}$ also noted that:

"Well, Big Data per se no, but data analysis that I understand I kind of the same in a much smaller scale, yes, I've seen the things that you can do by analysing data, things like, create predictions and improve the decision-making process, I think that the benefits of BD would be something similar, right?".

The above indicates that even when part of the industry recognises and understand the benefits or implementation of $\mathrm{BD}$ in other industry the need still exist to expand the knowledge and strengthen the relationship with the construction industry, by promoting the use of the technology and including these examples as part of the motivation for the implementation.

Ultimately, the remaining 10\% (2 out of 21) of interviewees demonstrated an advanced level of awareness with very specific examples of implementation outside construction, which places them at level 3 of Endsley's model. For instance, Interviewee $\mathrm{I}_{21}$ said that:

"Yes, according to what I learned it has been developed or used in areas such as health to predict outbreaks by analysing the behaviour of previous outbreaks. And to predict changes in retail, where they can predict what the clients will buy by also analysing their purchase behaviour and that way, they can send you personalised ads in your computer. But of course, it raises some questions about security and the management of the data that we allow some apps and companies to have about us".

The low percentage of the industry which possesses this type of essential knowledge demonstrates the need to educate current and future professionals in areas that could be projected as the future of the industry, its inclusion in the educational curriculum and professional training is evidenced by the lack of generalized knowledge and understanding that is demonstrated in each of the questions above. The industry needs to modernize, starting with the recognition of practices that are being currently implemented and that in the near future can help the adoption of new technologies such as Big Data.

\section{Discussion}

The need to manage increasing data volumes produced today is the major key driver for many industries that have already been making inroads in the adoption of Big Data and data management technology for years (Chen et. al. and Tamiminia et. al., 2020), a phenomenon that undoubtedly also occurs within the construction industry. Plenty 
DOI: $10.1108 / C I-05-2021-0090$

of literature exists reflecting on the positive results of adoption BD in other disciplines (Tamiminia et al., 2020; Caesarius and Hohenthal, 2017; Pigni, Piccoli, and Watson, 2016; The Economist, 2012; Raguseo, 2020), while limited sources exist about Big Data adoption within the construction industry indicating the presence of a gap.

This study represents the first step towards the adoption of Big Data within the construction in which specific case could positively impact the achievement of some of the Sustainable Development Goals in the most specific case of the strategies linked to the construction industry. The study was carried out by assessing the level of awareness that the main characters, on which a future BD implementation in construction will depend, possess of the topic. The results revealed the awareness state of the industry when it comes to Big Data, showing overall, that there is a basic level of awareness prevailing within this field, indicating that a primary step for the implementation of BD should be the popularization of the term and its characteristics. This is also supported by the high level of ignorance around BD requirements or characteristics, which is also reflected in the results. This indicates that these requirements and more technical aspects of this technology together with the concept should reach a higher level of generalisation to ensure a better understanding of the technology and its requirements.

Surprisingly, the results demonstrated that both the concept of big data and its characteristics are known more widely within the industry (Section 5.1), which agrees with much of the literature indicating that in recent years there has been an exponential increase in the use of both the term and the technology (Wang et. al., 2020). On the contrary, the study shows that the depth of this knowledge is still very little, evidenced by the need to further explore not only the concept but all aspects of this technology including its application in other industries which could be translated in a better general understanding of the technology (Section 5.4). Moreover, the basic elements for data accumulation are either not known or not implemented within the construction companies, or in some other cases known but not recognised as such by the decision-makers (Section 5.3). The study also showed that the industry has the desire and the ability to invest in new technologies which can demonstrate a positive impact in production and management, but it is faced with the barrier of lack of understanding and implementation of the elements necessary for its execution.

The accumulation of large volumes of data is the main driver for the implementation of BD in most industries (Boyd and Crawford, 2012), but the inherent resistance of the construction industry to implement new technologies that do not have a high and proven success factor plays against any move towards implementation (Ngo, et. al., 2020), as indicated by the need for more companies that take risks to adopt new technologies and serve as an example for adoption (Section 5.3), reflected in the results. Thus, indicating that overcoming this is a primary aspect to develop for successful adoption of Big Data and any new technology. In the same line, the results also showed that the construction industry has lagged in investing in tools that allow it to manage data more efficiently and in accordance with the requirements of current technology.

The construction industry fails to recognise the high volume of data produced as the asset other industries are already taking advantage of (Chen et. al., 2020 and Caesarius and Hohenthal, 2017) which needs to be addressed by providing development programs for present and future professionals of the industry, thus ensuring the creation of bold leadership who are not afraid to take risks nor to explore areas that are projected as the future of the industry (sections 5.1 and 5.4).

The lack of in-depth and homogenous understanding appears to be an issue that came to light in each area explored by this research for which it could be considered as the main problem, followed by the need for education and training aimed at present and future professionals to equip them with the tools that allow them to identify, take advantage of and implement technologies that will positively impact the industry in the future. Also, more insightful and risk-taker leadership is needed to guide the industry through the impending changes that await on the horizon, new technologies are being developed every day so the industry needs to be ready to learn and implement those that can produce positive changes and that can help to keep up with the changing needs of today and tomorrow's society. Ultimately, for the adoption of this technology that could immensely impact the future development of the industry, more understanding, education, examples, and individuals who risk being the first to exploit their potential are needed.

The findings of this research will allow researchers and managers to be aware of the potentials of this technology, of what is needed to take advantage of it and at the same time, understand that this is a turning point for the industry in which it is necessary to pivot and abandon old beliefs and accept change, embrace modernization and start preparing for industrial digitalisation. This could potentially help to react accordingly and to create a new learning environment to minimize those obstacles at the time of implementation. 


\section{Conclusion}

The adoption of Big Data has become a primary goal for most industries across the world, driven by the exponential increase in data generation and the proliferation of data-generating elements that seek to facilitate the management of operations both within companies and in daily life. In the same way, it has been shown that the construction industry, on many occasions, lags in the adoption of new technological developments such as BD. While the creation of large volumes of data that have led to the expansion of this technology through different industries will continue to grow along with technological developments, thus industries like construction must take advantage of the opportunities offered by this type of tools.

As a first step towards the adoption of BD in construction, this study sought to determine the level of awareness about BD that representatives within the decision-making level of construction companies possess, to identify the strategies that could facilitate future implementation of the technology across the whole sector. Situation awareness was selected since it is a main element of the decision-making process. The assessment of the level of awareness of BD served to identify the strategies that could facilitate a path for industry-wide implementation.

The data analysis was carried out applying a modification of the Endsley model, which served to qualify the answers obtained in the semi-structured interview. Four levels of awareness were identified ranging from level 0 or no awareness to level 3 or advanced awareness. The results showed that although there is a relatively high level of knowledge about the BD concept and its characteristics as well as a high level of willingness to productivityenhancing technologies, the depth of that knowledge is not nearly enough to start the general adoption of the technology. Also, to this, we must add the high level of ignorance about the basic steps of data management and $\mathrm{BD}$ applications in other industries. The first is important because it is the foundation of the dataset creation necessary to promote a BD analysis, and the latter because it could boost the interest of investors and start an industry-wide implementation of the BD technology.

This paper provides a richer insight into the understanding of awareness and how it could be used to explore topics related to the implementation of new technologies. This study contributes towards the understanding of what are the first steps towards Big Data adoption and provides a source that can be used to inform current and future professionals about the technologies that will certainly be relevant in the near future of the construction industry.

Many needs could be identified as a result of this study and strategies were provided to meet these needs such as the promotion and understanding of the concept and its characteristics, the need to provide training and education to the professionals who lead the industry in the future, to promote the use and applications of the technology not only in the construction industries but also in other areas that could serve as an example, providing this way to improve general understanding, among others, many of the strategies presented in this study will serve not only for the future adoption of BD but any technology-related change that could be introduced in the future, these examples can be applied as well outside the Dominican Republic, in countries that share an industry attached to the "traditional" practices that have been used for many years.

This study could be continued by exploring other barriers, challenges, key motivators and opportunities presented by big data adoption, taking other industries as an example. Future studies could also explore the level of awareness in other settings like more developed countries in order to determine if this factor influences the adoption of new technologies. In the same way, a study of the financial performances of companies that have implemented BD across all industries could demonstrate if the BD benefits vary depending on the type of organisation.

This study is limited to analysing concepts related to general knowledge about Big Data, and its results apply within the framework of the construction industry of the Dominican Republic, although the results may apply to other countries with similar conditions, as mentioned earlier. Other key aspects for implementation such as exploration within the framework of other countries can be used as the objectives of future research.

Finally, the implications of this study are to the professional involved in the digital process and decision-makers in the construction industry. Also, the construction education sector which aims to provide educational tools to better prepare future professionals, in addition, to provide continuous improvement training to achieve a standardised level of awareness of BD for the current construction professionals.

\section{References}

Bilal, M., Oyedele, L.O., Qadir, J., Munir, K., Ajayi, S.O., Akinade, O.O., Owolabi, H.A., Alaka, H.A., Pasha, M., Big Data in the construction industry: a review of present status, opportunities, and future trends, Adv. Eng. 
Inform. 30 (2016) 500-521, https://doi.org/10.1016/j.aei.2016.07.001.

Boyd, D. and Crawford, K., 2012. Critical questions for big data: Provocations for a cultural, technological, and scholarly phenomenon. Information, communication \& society, 15(5), pp.662-679.

Caesarius, L.M. and Hohenthal, J. (2018) Searching for big data. Scandinavian Journal of Management [online], 34(2), pp. 129-140 Available at:<http://dx.doi.org/10.1016/j.scaman.2017.12.002>.

Caulfield, J. (2019) How to do thematic analysis6 of September-last update [Accessed 28 May 2020]. Available at: <https://www.scribbr.com/methodology/thematic-analysis/>.

Central Bank of the Dominican Republic (2020), "Gross Domestic Product (GDP) by the spending approach. Current values and chained volume indices referenced to 2007-2019, quarterly. March 2020”

Chen, P., Linc, C. and Wud, W. (2020) Big Data Management in healthcare: Adoption challenges and implications. International Journal of Information Management [online], 26(1), pp. 1-2 Available at: $<$ http://dx.doi.org/10.1016/j.ijinfomgt.2005.10.001 >.

Choi, M., Ahn, S. and Seo, J. (2020) VR-Based investigation of forklift operator situation awareness for preventing collision accidents available at:

$<$ http://www.sciencedirect.com/science/article/pii/S0001457519308346>.

Cohen D, Crabtree B. "Qualitative Research Guidelines Project." July 2006. http://www.qualres.org/HomeSemi3629.html

Creswell, J.W. (2013), Research Design: Qualitative, Quantitative, and Mixed Methods Approaches, Sage publications, Thousand Oaks, CA.

Crouch, M., \& McKenzie, H. (2006). The logic of small samples in interview-based qualitative research. Social Science Information, 45(4), 18. DOI: 10.1177/0539018406069584

DeJonckheere, M. and Vaughn, L. M. (2019) 'Semistructured interviewing in primary care research: a balance of relationship and rigour', Family Medicine and Community Health, 7(2), p. e000057. DOI: 10.1136/fmch-2018000057.

Dishman, D., Fallacaro, M.D., Damico, N. and Wright, M.C. (2020) Adaptation and Validation of the Situation Awareness Global Assessment Technique for Nurse Anesthesia Graduate StudentsAvailable at: $<$ http://www.sciencedirect.com/science/article/pii/S1876139920300141>.

Dlamini, S. (2012) Relationship of construction sector to economic growth. School of Construction Management [online].

Endsley, M.R., Measurement of situation awareness in dynamic environments Human Factors, 37 (1) (1995), pp. $65-84,10.1518 / 001872095779049543$

Endsley, M.R. (2014) Situation Awareness. in Zsambok,C. E. and Klein, G.(eds). Naturalistic Decision Making. New York, USA: Psychology Press, pp.269-284.

Filipović, D., Lukić, A. and Lukić, M. (2020) geospatial evaluation of belgrade for the purposes of determination of suitable locations for the construction of pv plants. Archives for Technical Sciences [online], 1(22), pp. 59-66.

Gandomi, A. and Haider, M. (2015) 'Beyond the hype: Big data concepts, methods, and analytics', International Journal of Information Management. Elsevier Ltd, 35(2), pp. 137-144. DOI: 10.1016/j.ijinfomgt.2014.10.007.

Guest, G., Bunce, A., \& Johnson, L. (2006). How many interviews are enough? An experiment with data saturation and variability. Field Methods, 18(1), 24. DOI: 10.1177/1525822X05279903

Khan, R. A. (2008) Role of Construction Sector in Economic Growth: Empirical Evidence from

Pakistan Economy.First International Conference on Construction In Developing Countries (ICCIDC-I). August 4-5, 2008. Karachi, Pakistan: Available at: $<$ https://www.researchgate.net/publication/283007781 $>$.

Kamunda, A., Renukappa, S., Suresh, S., and Jallow, H., (2020) BIM in the Water Industry: Addressing 
Challenges to improve the project delivery process, Engineering, Construction and Architectural Management (ECAM), Special issue: Construction 4.0, Emerald publishing, DOI: 10.1108/ECAM-12-2019-0692.

Khurshid, M.M., Zakaria, N.H., Rashid, A., Kazmi, R., Shafique, M.N. and Ahmad, M.N. (2019) Analyzing diffusion patterns of big open data as policy innovation in public sector. Computers and Electrical Engineering [online], 78pp. 148-161 Available at: $<$ http://dx.doi.org/10.1016/j.compeleceng.2019.07.010>.

Kumar, R. (2014) Research Methodology: A Step-by-Step Guide for Beginners. 4th Edition, SAGE Publications Ltd., London.

Latham, J. (2020) Qualitative Sample Size - How Many Participants is Enough? Available at $<$ https://www.drjohnlatham.com/many-participants-enough/>.

Liu, P., 2015. A survey of remote-sensing big data. Front. Environ. Sci. 3, 45.

Li, S., Dragicevic, S., Castro, F.A., Sester, M., Winter, S., Coltekin, A., Pettit, C., Jiang, B., Haworth, J., Stein, A. and Cheng, T. (2016) Geospatial big data handling theory and methods: A review and research challenges. ISPRS Journal of Photogrammetry and Remote Sensing [online], 115pp. 119-133 Available at: $<$ http://dx.doi.org/10.1016/j.isprsjprs.2015.10.012>.

MEPYD Cuarto Informe Anual de Avance en la Implementacion de la Estrategia Nacional de Desarrollo 2030 y Cumplimiento de los Objetivos y Metas del Plan Plurianual del Sector Publico 2015, August. Santo Domingo, República Dominicana: Ministerio De Economía, Planificación Y Desarrollo. May 2017

Mason, S. (2020) Practice makes better? Testing a model for training program evaluators in situation awareness, available at: <http://www.sciencedirect.com/science/article/pii/S0149718919303295>.

McCombes, S. (2021) Sampling Methods | Types and Techniques Explained, Scribbr. Available at: https://www.scribbr.com/methodology/sampling-methods/ (Accessed: 2 May 2021).

Moura, I., Teles, A., Silva, F., Viana, D., Coutinho, L., Barros, F. and Endler, M. (2020) Mental health ubiquitous monitoring supported by social situation awareness: A systematic review, available at:

$<$ http://www.sciencedirect.com/science/article/pii/S1532046420300824>.

National Congress of the Dominican Republic (2008), 'Law No. 488-08”, National Congress of the Dominican Republic, Santo Domingo.

Naoui, M. A. et al. (2020) 'Using a distributed deep learning algorithm for analyzing big data in smart cities', Smart and Sustainable Built Environment, 10(1), pp. 90-105. DOI: 10.1108/SASBE-04-2019-0040.

Neilson, A., Indratmo, Daniel, B. and Tjandra, S. (2019) Systematic Review of the Literature on Big Data in the Transportation Domain: Concepts and Applications. Big Data Research [online], 17pp. 35-44 Available at: $<$ http://dx.doi.org/10.1016/j.bdr.2019.03.001>.

Ngo, J., Hwang, B. and Zhang, C. (2020) Factor-based big data and predictive analytics capability assessment tool for the construction industry, available at:

$<$ http://www.sciencedirect.com/science/article/pii/S0926580519306818>.

Oudjehane, A., Moeini, S., Big Data in Construction Projects: Risk and Opportunity Management, http://pmsymposium.umd.edu/pm2017/wp- content/uploads/sites/

3/2017/01/Big_Data_in_Construction_Projects_Risk_and_Opportunity_Management-Moeini.pdf, (2017), Accessed date: 11 June 2019.

Pigni, F., Piccoli, G., \& Watson, R. (2016). Digital data streams: Creating value from the real-time flow of big data. California Management Review, 58(3), 5-25.

Polit, D.F. and Beck, C.T. (2010) Generalization in quantitative and qualitative research: Myths and strategies, available at: <http://www.sciencedirect.com/science/article/pii/S0020748910002063>.

Rabhi, L., Falih, N., Afraites, A. and Bouikhalene, B. (2019) Big Data Approach and its applications in Various Fields: Review. Procedia Computer Science [online], 155pp. 599-605 Available 
at: $<$ http://dx.doi.org/10.1016/j.procs.2019.08.084>.

Raguseo, E. (2017) Big data technologies: An empirical investigation on their adoption, benefits

and risks for companies. International Journal of Information Management [online], 26(1), pp. 187-195

Available at: $<$ http://dx.doi.org/10.1016/j.ijinfomgt.2005.10.001>.

RICS (2017) Big Data, Smart Cities and Intelligent Buildings. Insight Paper, The Royal Institution of Chartered Surveyors Publications, London,

https://www.rics.org/contentassets/cb3 fd55b63df4d1fa6b9d8e921a8ddfd/rics_smart_cities_big_data_2017_resea rch_110917_rt.pdf, (accessed, June 2020).

RICS (2020) The futures report, 2020, The Royal Institution of Chartered Surveyors, London, https://www.rics.org/globalassets/rics-website/media/news/news--opinion/rics-future-report-2.pdf, (accessed, June 2020).

Sarkar, D. and Modi, R., 2015. Applications of building information modelling (BIM) to real estate projects of Ahmedabad. International Advanced Research Journal in Science, Engineering and Technology, 2(9), pp.54-58.

Sayah, Z. et al. (2020) 'An intelligent system for energy management in smart cities based on big data and ontology’, Smart and Sustainable Built Environment. DOI: 10.1108/SASBE-07-2019-0087.

Silverio-Fernandez, M., Renukappa, S., and Suresh, S., (2019), Evaluating critical success factors for implementing smart devices in the construction industry: An empirical study in the Dominican Republic, Engineering, Construction and Architectural Management, Emerald publishing, Vol. 26 Issue: 8, pp.1625-1640, DOI: 10.1108/ECAM-02-2018-0085.

Silverio Rodriguez, 2020, "Implementation of Building Information Modelling in The Dominican Republic Construction Industry”, Doctor in Philosophy, University of Wolverhampton, Wolverhampton.

Tabesh, P., Mousavidin, E. and Hasani, S. (2019) Implementing big data strategies: Amanagerial perspective. Business Horizons [online], 62(3), pp. 347-358 Available at: $<$ http://dx.doi.org/10.1016/j.bushor.2019.02.001>.

Tamiminia, H., Salehi, B., Mahdianpari, M., Quackenbush, L., Adeli, S. and Brisco, B. (2020) Google Earth Engine for geo-big data applications: A meta-analysis and systematic review. ISPRS Journal of Photogrammetry and Remote Sensing [online], 164pp. 152-170 Available at:<http://dx.doi.org/10.1016/j.isprsjprs.2020.04.001>.

The Economist (2012). Big data: Crunching the numbers. Special report on international banking May 19.

The World Bank (2020), "World Bank open data", available at: https://data.worldbank.org/(accessed June 2020).

The World Bank (2021), 'República Dominicana: panorama general”, available at:

https://www.worldbank.org/en/country/dominicanrepublic/overview\#1 (accessed June 2021).

Wang, Y., Wang, S., Yang, B., Zhu, L. and Liu, F. (2020) Big data driven Hierarchical Digital Twin Predictive Remanufacturing paradigm: Architecture, control mechanism, application scenario and benefits. Journal of Cleaner Production [online], 248pp. 119299 Available at:<http://dx.doi.org/10.1016/j.jclepro.2019.119299>. 